UCRL-JC-118004

PREPRINT

\title{
Ultra-Thin Carbon Coatings for Head-Disk Interface Tribology
}

\author{
J.N. Glosli \\ J. Belak \\ M.R. Philpott
}

This paper was prepared for submittal to the

Materials Research Society Conference

San Francisco, $C A$

April 17-21, 1995

January 1995

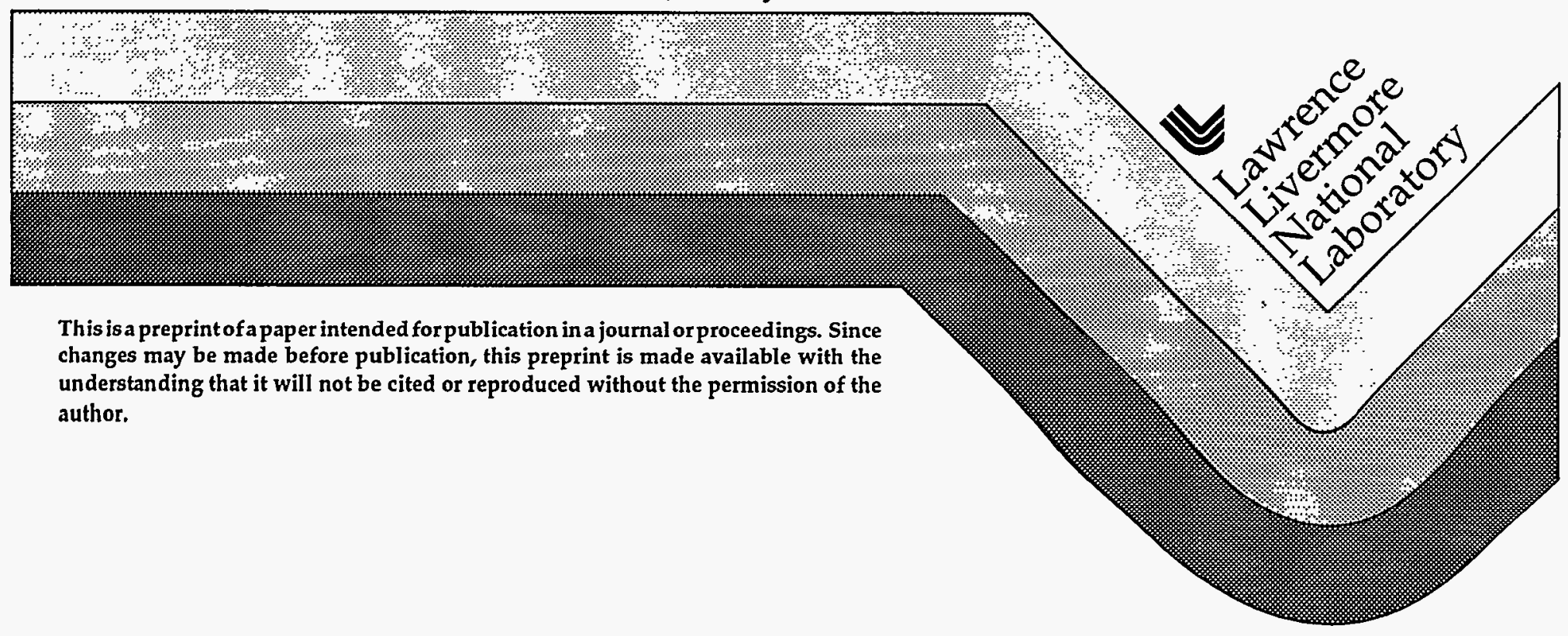




\section{DISCLAIMER}

This document was prepared as an account of work sponsored by an agency of the United States Government. Neither the United States Government nor the University of California nor any of their employees, makes any warranty, express or implied, or assumes any legal liability or responsibility for the accuracy, completeness, or usefulness of any information, apparatus, product, or process disclosed, or represents that its use would not infringe privately owned rights. Reference hercin to any specific cornmercial products, process, or service by trade name, trademark, manufacturer, or otherwise, does not necessarily constitute or imply its endorsement, recommendation, or favoring by the United States Government or the University of California. The views and opinions of authors expressed herein do not necessarily state or rellect those of the United States Government or the University of Calfforniz, and shall not be used for advertising or product endorsement purposes. 


\section{DISCLAIMER}

Portions of this document may be illegible in electronic image products. Images are produced from the best available original document. 
J.N. GLOSLI* J. BELAK ${ }^{*}$, and M.R. PHILPOTT ${ }^{* *}$

*University of California, Lawrence Livermore National Laboratory, Livermore, CA 94550

*IBM Research Division, Almaden Research Center, 650 Harry Road, San Jose, CA 95120-6099

\section{ABSTRACT}

Molecular dynamics computer simulations of the growth processes and microstructural properties of amorphous carbon $(\mathrm{a}: \mathrm{C})$ and amorphous hydrogenated carbon $(\mathrm{a}: \mathrm{CH})$ ultra-thin films have been performed. Films 1 to $10 \mathrm{~nm}$ thick were grown on a diamond (100) surface using Brenner's[1-2] bond-order potential for hydrocarbons. The stoichiometry, radial distribution function, chemical bonding (amount of $\mathrm{sp}^{2}$ and $\mathrm{sp}^{3}$ hybridization) and residual stress are presented.

\section{INTRODUCTION}

Amorphous carbon films approximately $20 \mathrm{~nm}$ thick are used as protective coatings on magnetic recording disks. As storage densities increase, the role of the overcoat becomes increasingly important because of smaller spacings between the recording head and the spinning disk. Furthermore, future generation disks call for an overcoat thickness of $5 \mathrm{~nm}$ or less. These small length scales and the high speed of the spinning disk $(10-30 \mathrm{~m} / \mathrm{s})$ suggest that a molecular dynamics (MD) model[3] might provide useful insight into friction and wear mechanisms when the head and disk make contact.

One of the necessary inputs required to carry out such an $\mathrm{MD}$ model is a specification of the position of all of the atoms in the simulation, i.e. a detailed specification of the material microstructure. Such a detailed understanding of the microstructure of amorphous carbon overcoats does not presently exist. Neutron[4] and electron[5] diffraction studies demonstrate that the material is amorphous. Previous classical MD simulations[6-8] yield pair distribution function in qualitative agreement with the diffraction studies, but they all differ in detail. More recent, quantum mechanical tight-binding $\mathrm{MD}$ (TBMD) studies[9-11] give a better description of the interatomic interactions and the chemical hybridization ( $\mathrm{sp}^{2}$-graphite-like versus $\mathrm{sp}^{3}$-diamond-like). However, these studies are presently limited to rather small system sizes and rapid quench rates.

In this paper we present both quench and deposition simulations of the formation of amorphous carbon using Brenner's[1-2] bond-order potential model. This classical potential mimics the quantum mechanics allowing carbon to form strong chemical bonds with a variety of hybridizations.

\section{METHODS and RESULTS}

We have performed two types of simulations: quench from a high temperature liquid and deposition onto a room temperature surface. During a quench simulation, we use periodic boundary conditions and constrain the volume to be $85 \%$ of diamond density $\left(\rho_{D}=3.54 \mathrm{gms} / \mathrm{cc}\right)$. The initial state is a well equilibrated liquid at $6000 \mathrm{~K}$. We have used system sizes ranging from 64 to 8192 carbon atoms and quench rates ranging from 5 to $40 \mathrm{~K} / \mathrm{ps}$. It was found that the system formed unphysical bonding configurations without an added torsional energy between $\mathrm{sp}^{2}$ hybrid- 
ized carbon atoms.[2] This torsional energy was included for all results presented here.

The pair distribution function, shown in

Figure 1, was found to be relatively insensitive

to quench rate and system size. The curve is

remarkably similar to the TBMD simulations,[9]

Though other properties that characterize the microstructure are different. The bonding character $\left(\mathrm{sp}^{2} / \mathrm{sp}^{3}\right)$ is sensitive to quench rate, with $10 \% \mathrm{sp}^{2}$ at $5 \mathrm{~K} / \mathrm{ps}$ and $16 \% \mathrm{sp}^{2}$ at $40 \mathrm{~K} / \mathrm{ps}$. The $\mathrm{sp}^{2}$ carbon atoms tend to form pairs, as in the TBMD simulations.[9-11] The sensitivity to quench rate is probably related to the time required to form these pairs and other extended structures from the initially homogeneous liquid. In the large system size quenches, we find large regions of $\mathrm{sp}^{3}$ bonding with small regions of $\mathrm{sp}^{2}$ bonding interdispersed. Further analysis is required to quantitatively relate these results to previous hypothesis.[12]

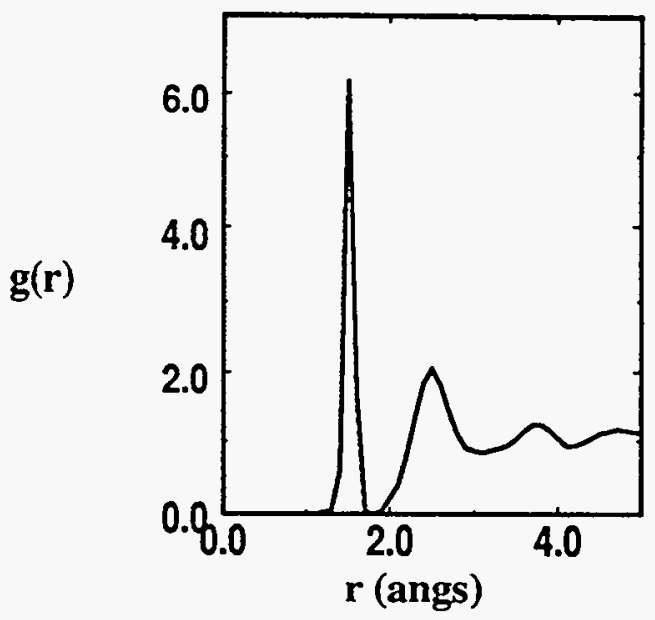

Figure 1. The calculated pair distribution function for a quench amorphous carbon using 8192 atoms and a quench rate for $5 \mathrm{~K} /$ ps.

More relevant for magnetic disk overcoats are films grown in a deposition processes. We start with a diamond (100) surface held at room temperature (the Brenner potential predicts a $2 \times 1$ reconstruction). Carbons atoms are rained down onto random surface positions at a rate of 1 atom/ ps with average kinetic energies ranging from $1 \mathrm{eV}$ to $100 \mathrm{eV}$. Faster deposition rates do not allow the thermostat sufficient time to remove the heat formed when the incident atoms form chemical bonds with the surface. Experimental deposition rates are many orders of magnitude slower than this and the surface is probably in equilibrium.

The results of three simulations $(5 \mathrm{eV}, 20 \mathrm{eV}$, and $100 \mathrm{eV})$ are shown in Figure 2 after several nanoseconds of simulation time (we use a time step of one femtosecond). Our computer simulation is open. That is, atoms and molecules that form in the vapor above the growing surface and migrate past the upper boundary are removed from the simulation. For this reason the exact time to grow a film of given thickness is unknown at the start of the simulation. The periodicity of the surface cell is about $2 \mathrm{~nm}$ and the films are about $4 \mathrm{~nm}$ thick. The microstructure of the films and the morphology of the growth process varies significantly with the deposition energy. At low energy, the film is at lower density $\left(\rho / \rho_{D} \sim 0.7\right)$ with a significant fraction $(70 \%)$ of $\mathrm{sp}^{2}$ hybridized carbon atoms. This is shown more clearly in the density profiles. There are large open regions in the film and chain-like $\left(\mathrm{sp}^{1}\right)$ structure growing on the surface. Increasing the energy to $20 \mathrm{eV}$, we find a very compact film at near diamond density $\left(\rho / \rho_{D} \sim 1\right)$ with only $10 \% \mathrm{sp}^{2}$ carbons in the film. The interface between the film and the vapor is very smooth. This interface becomes very diffuse at high energy $(100 \mathrm{eV})$ where many carbon atoms leave through the vapor. The resulting film is less dense $\left(\rho / \rho_{D} \sim 0.85\right)$ though not containing the open regions found at low energy. The $\mathrm{sp}^{2}$ fraction has increased to about $20 \%$.

The density profiles for films grown with hydrogen are shown in Figure 3. Here we deposit an equal concentration of carbon and hydrogen atoms, though only $20-30 \%$ of the atoms in the films are hydrogens. The rest of the hydrogen atoms leave through the vapor as small hydrocarbon molecules. The presence of hydrogen lowers the number of $\mathrm{sp}^{2}$ carbons at low energy and increases the number at high energy. The most pronounced effect of the hydrogen, however, is to lower the stress in the films. This is shown in Figure 4 for both pure carbon and hydrogenated carbon films with deposition energy ranging from $5-100 \mathrm{eV}$. At $20 \mathrm{eV}$ the stress in the hydrogenated film is 4 times smaller than for the pure carbon film at the same deposition 


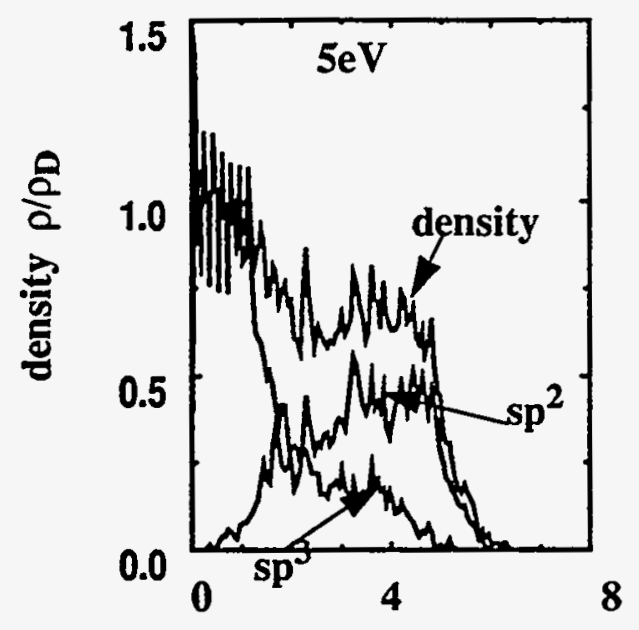

(a)

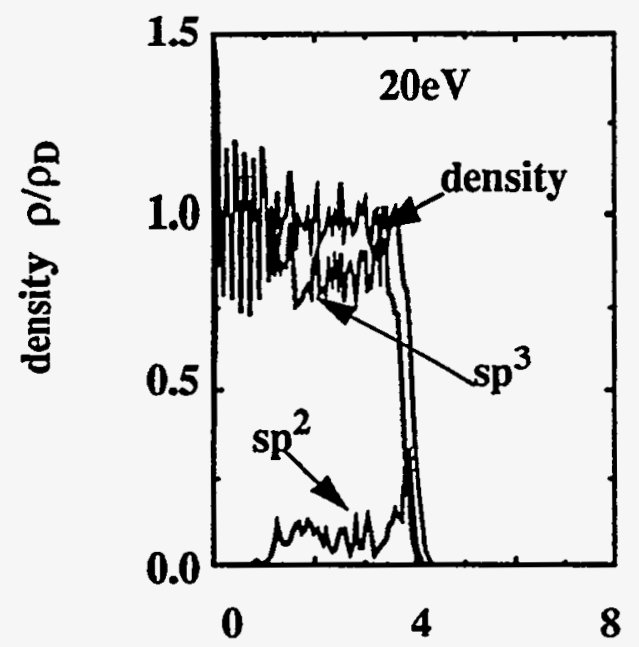

(b)
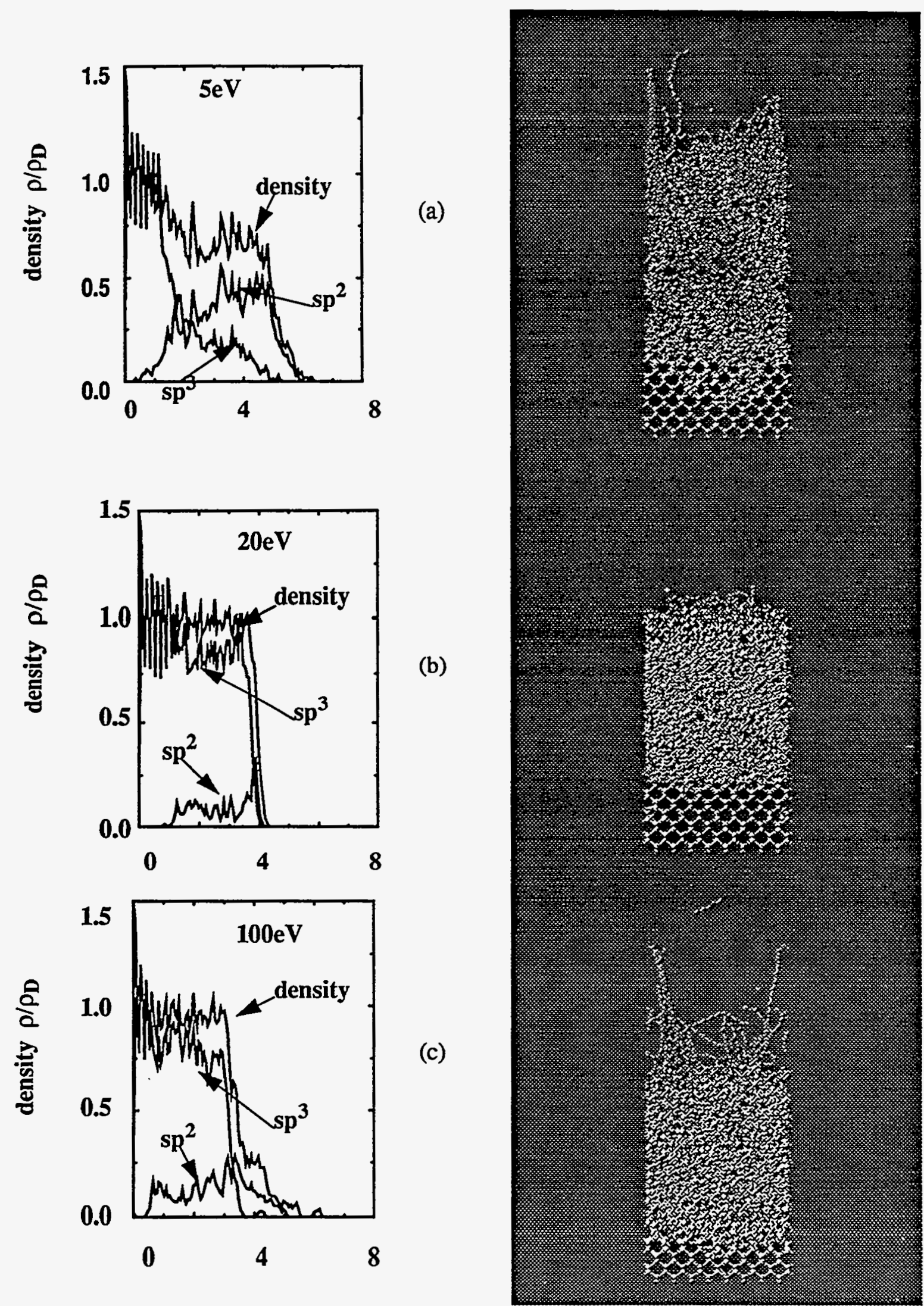

Figure 2. The growth of amorphous carbon films (a:C) on diamond (100) at deposition energies (a) $5 \mathrm{eV}$, (b) $20 \mathrm{eV}$, and (c) $100 \mathrm{eV}$. Density profiles are normalized by diamond density and are shown as a function of height through the film (in nm). 

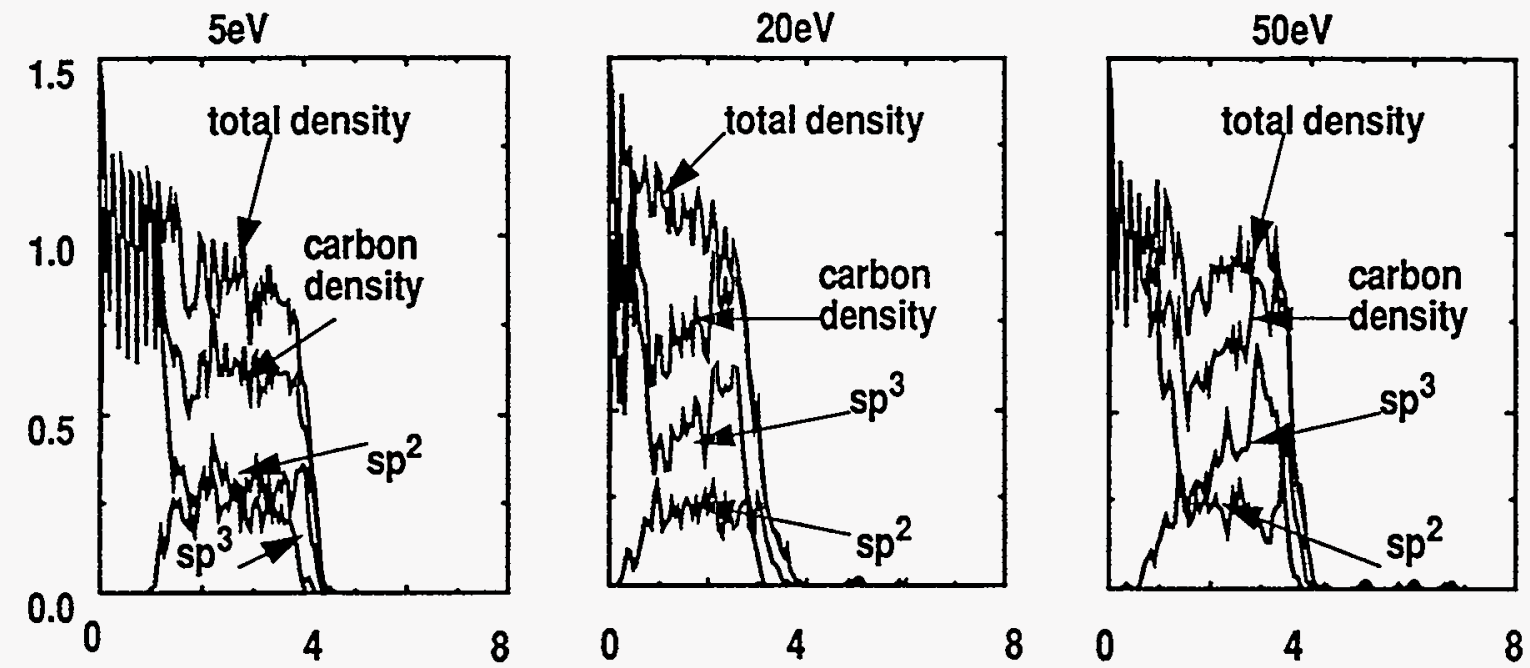

Figure 3. The density profiles (normalized by the density of diamond) in simulated amorphous hydrogenated carbon films (a:CH) at deposition energies of $5 \mathrm{eV}, 20 \mathrm{eV}$, and $50 \mathrm{eV}$ as a function of position in the film in $\mathrm{nm}$. The 4 curves are the total density, the density of all forms of carbon, the density of $\mathrm{sp}^{2}$ carbon and the density of $\mathrm{sp}^{3}$ carbon.

energy. The values of the stress are qualitatively in agreement with experiment though we note that the small size of our simulation cell inhibits large scale relaxation mechanisms. Large surface cells need to be simulated to further quantify the results.

\section{DISCUSSION}

We have shown that classical molecular dynamics simulations using Brenner's bondorder potential model with additional torsional energy terms give quantitative agreement with
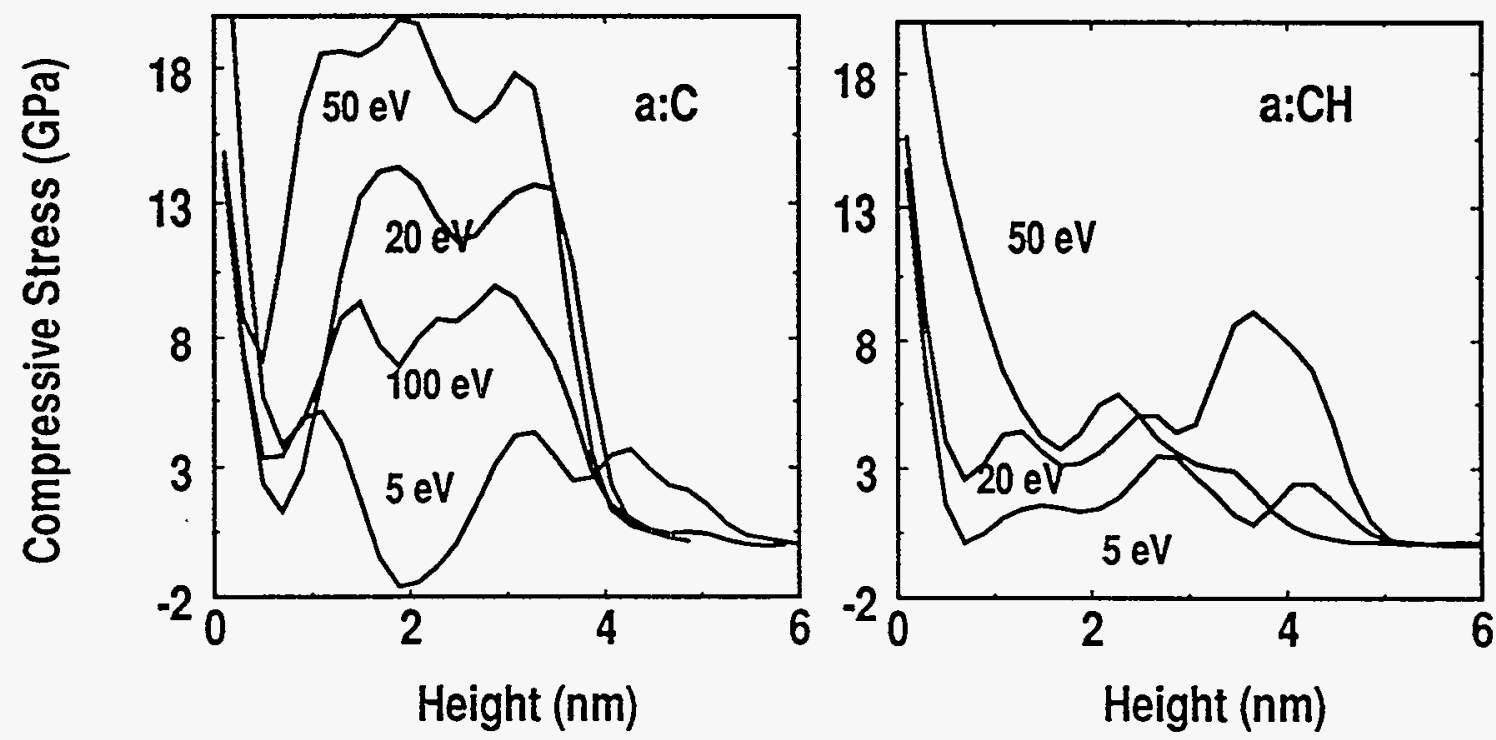

Figure 4. Residual stress (in $\mathrm{GPa}$ ) as a function of height (in $\mathrm{nm}$ ) for $\mathrm{a}: \mathrm{C}$ and $\mathrm{a}: \mathrm{CH}$ films grown at deposition energies of $5 \mathrm{eV}, 20 \mathrm{eV}, 50 \mathrm{eV}$ and $100 \mathrm{eV}$. 
tight-binding molecular dynamics for the microstructure of quenched amorphous carbon films. For a 64 atom quench we find $56 \mathrm{sp}^{3}$ and $8 \mathrm{sp}^{2}$ carbons, 6 of which come in pairs. The recent TBMD of Drabold, et al.[11] found $6 \mathrm{sp}^{2}$ carbons, all of which were paired. It appears the potential gives a reasonable description of the energy surface for carbon covalent bonding, though we do not know to what extend the kinetics (or transition states) are correct.

The deposition simulations show some interesting features. Hydrogen increases the $\mathrm{sp}^{3}$ bonding of films grown at low energy and plays an important role in releasing residual stress in the films. The stress in our simulated films reaches a maximum of about $18 \mathrm{GPa}$ for pure carbon at a deposition energy of 50eV. Experiments[5] for deposition of very high carbon concentration show a maximum stress of about $8 \mathrm{GPa}$ at a deposition energy of $20 \mathrm{eV}$. The discrepancy is most likely due to the pure carbon in our simulation (no impurities) and the small size of our simulation cell. Both of which will lower the stress. The density is maximal near the deposition energies (20$40 \mathrm{eV}$ ) used to grow films on magnetic disks. We might expect these films with high density and lower stress to have good tribological properties. Another interesting aspect of the simulations is the thickness of the interface between the growing film and the vapor above. This thickness is large (several nanometers) at both low and high deposition energy and minimal at moderate deposition energy $(20 \mathrm{eV})$. With the trend towards thinner overcoats on magnetic disks $(5 \mathrm{~nm})$, the properties of the overcoat may be dominated by interfacial phenomena. In the future, we plan to grow films on a larger surface unit cell and perform MD simulations of friction and wear.

\section{ACKNOWLEDGEMENTS}

Work performed under the auspices of the U.S. Department of Energy by the Lawrence Livermore National Laboratory under contract No. W-7405-ENG-48.

\section{REFERENCES}

1. D.W. Brenner, Phys. Rev. B 42, 9458 (1990).

2. D.W. Brenner, J.A. Harrison, C.T. White, and R.J. Colton, Thin Solid Films 206, 220 (1991).

3. M.P. Allen and D.J. Tildesley, Computer Simulation of Liquids (Oxford, Clarendon, 1987).

4. F. Li and J.S. Lannin, Phys. Rev. Lett. 65, 1905 (1990).

5. D.R. McKenzie, D. Muller, and B.A. Pailthorpe, Phys. Rev. Lett. 67, 773 (1991).

6. J. Tersoff, Phys. Rev. Lett. 61, 2879 (1988).

7. B.A. Pailthorpe, J. Appl. Phys. 70, 543 (1991).

8. H.-P. Kaukonen and R.M. Nieminen, Phys. Rev. Lett. 68, 620 (1992).

9. C.Z. Wang, K.M. Ho, and C.T. Chan, Phys. Rev. Lett. 70, 611 (1993).

10 C.Z. Wang and K.M. Ho, Phys. Rev. Lett, 71, 1184 (1993).

11. D.A. Drabold, P.A. Fedders, and P. Stumm, Phys. Rev. B 49, 16415 (1994).

12. J. Robertson and E.P. O'Reilly, Phys. Rev. B 35, 2946 (1987). 